\title{
Biodegradable Polymer-Based Sirolimus Coating Stent in a Porcine Coronary Restenosis Model
}

\author{
Kyung Seob Lim, ${ }^{1,2,3 *}$ Jun-Kyu Park, ${ }_{1}^{5 *}$ Myung Ho Jeong, ${ }^{1,2,3}+$ In Ho Bae, ${ }^{1,2}$ Jae-Woon Nah, ${ }^{5}$ Dae Sung Park, ${ }^{2,3}$ Jong Min Kim, ${ }^{2,3}$ Jung Ha Kim, ${ }^{2,3}$ So Yeon Lee, \\ Eun Jae Jang, ${ }^{1,2}$ Nan Yeol Kim, ${ }^{2,3}$ Doo Sun Sim, ${ }^{2,3}$ Keun-Ho Park, ${ }^{2,3}$ Young Joon Hong, ${ }^{2,3}$ Youngkeun Ahn, ${ }^{2,3}$ Jung Chaee Kang ${ }^{2,3}$ \\ ${ }^{1}$ Korea Cardiovascular Stent Institute, Jangsung; \\ ${ }^{2}$ Cardiovascular Convergence Research Center Nominated by Korea Ministry of Health and Welfare, Gwangju; \\ ${ }^{3}$ Cardiovascular Research Center, Chonnam National University Hospital, Gwangju; \\ ${ }^{4}$ Regeneromics Research Center, Chonnam National University, Gwangju; \\ ${ }^{5}$ Department of Polymer Science and Engineering, Sunchon National University, Sunchon, Korea
}

Received: 15 October 2014

Revised: 28 October 2014

Accepted: 28 October 2014

†Corresponding author:

Myung Ho Jeong, MD

Director of Korea, Cardiovascular Stent

Research Institute, Director of

Cardiovascular Convergence Research

Center Nominated by Korea Ministry of

Health and welfare, Director of

Department of Education and Research,

Chonnam National University Hospital,

671 Jaebong-ro, Dong-gu, Gwangju

501-757, Korea

Tel: +82-62-220-6243

Fax: +82-62-228-7174

E-mail:myungho@chollian.net

*Kyung Seob Lim and Jun-Kyu Park contributed equally to this work.

Copyright (C) Korean Society on Thrombosis and Hemostasis. All rights reserved.
Purpose: Biolimus A9 is a highly lipophilic derivative of sirolimus. The aim of this study was to compare a sirolimus- and a biolimus A9-eluting stent using the same biodegradable polymer (PLA, poly-L-lactic acid) in a porcine coronary overstretch restenosis model.

Methods: Pigs were randomized into three groups in which the coronary arteries (15 pigs, 10 coronaries in each group) had either sirolimus-eluting stents with PLA (SEP, $n=10$ ), biolimus A9-eluting stents with PLA (BEP, $n=10)$, or PLA-coated stents (PCS, $n=10$ ). Histopathologic analysis was performed at 28 days after stenting.

Results: In the release profiles, approximately $40 \%$ of the sirolimus and $50 \%$ of the biolimus A9 were released from the stent for 32 days. There were no significant differences in the injury score and the internal elastic lamina. Among the three groups, there were significant differences in the lumen area $(3.2 \pm 0.91$ $\mathrm{mm}^{2}$ in SEP vs. $3.1 \pm 1.10 \mathrm{~mm}^{2}$ in BEP vs. $0.5 \pm 0.56 \mathrm{~mm}^{2}$ in PCS, $\left.P<0.0001\right)$, in the neointimal area $(1.7 \pm 0.56$ $\mathrm{mm}^{2}$ in SEP vs. $1.9 \pm 0.95 \mathrm{~mm}^{2}$ in BEP vs. $4.3 \pm 0.63 \mathrm{~mm}^{2}$ in PCS, $P<0.0001$ ), in the percent area of stenosis $(35.2 \pm 13.77 \%$ in SEP vs. $39.0 \pm 20.00 \%$ in BEP vs. $89.4 \pm 10.92 \%$ in PCS, $P<0.0001)$, in the fibrin score $[2.0$ (range 1.0 to 3.0) in SEP vs. 2.0 (range 2.0 to 3.0) in BEP vs. 1.0 (range 1.0 to 2.0) in PCS, $P<0.0001$ ], and in the inflammation score [1.0 (range 1.0 to 2.0) in SEP vs. 1.0 (range 1.0 to 3.0) in BEP vs. 3.0 (range 2.0 to 3.0 ) in PCS, $P<0.0001]$. The percent area of stenosis using micro-computed tomography demonstrated similar restenosis rates with histopathological stenosis between SEP and BEP $(36.7 \pm 13.77 \%$ in SEP vs. $41.2 \pm$ $20.00 \%$ in BEP vs. $86.5 \pm 10.92 \%$ in PCS).

Conclusion: SEP and BEP showed a superior neointima suppressive effect compared to PCS at one month after stenting in a porcine coronary restenosis model. Therefore, sirolimus with biodegradable polymers can be a useful and selective drug for coating the coronary stent to inhibit neointimal hyperplasia.

Keywords: Stents, Percutaneous coronary intervention, Restenosis, Inflammation

\section{Introduction}

Durable polymer-based first-generation sirolimus-eluting stents (SES) reduce the risks of in-stent restenosis (ISR) and revascularization compared to bare metal stents (BMS)., ${ }^{1,2}$ Therefore, drug eluting stents (DES), such as those using sirolimus and paclitaxel, have increasingly become available and used for the treatment of atherosclerotic coronary artery stenosis lesions. However, the permanent polymer in these DES can cause chronic inflammation and hypersensitive reactions in the stented artery after drug release. This may lead to delayed re-endothelialization, delayed arterial healing, and late stent thrombosis. ${ }^{3-5}$

In order to overcome the shortcomings of durable polymers, new DES using biodegradable polymers have been developed. ${ }^{6}$ DES with biodegradable polymers have been shown to be safe and effective in clinical trials. ${ }^{7,8}$ For example, biolimus A9-eluting stents (BES, Biomatrix, Biosensors Interventional Technologies Pte Ltd., Singapore) were developed as a third-generation drug eluting stent containing a bioabsorbadable polymer (PLA, poly-L-lactic acid) with the highly lipophilic sirolimus derivative biolimus A9.

The objective of the present study was to evaluate a biodegradable polymer-based sirolimus coating on a novel bare-metal stent in a porcine coronary restenosis model.

\section{Methods}

Animal preparation and stent implantation

The animal study was approved by the Ethics Committee of Chonnam National University Medical School and Chonnam National University Hospital (CNU IACUC-H-2013-36), and conformed to the Guide for the Care and Use of Laboratory Animals published by the US National Institutes of Health (NIH Publication No. 85-23, revised 1996). Study animals were castrated male pigs weighing 20$25 \mathrm{~kg}$. To prevent acute thrombosis after stenting, premedication with aspirin $100 \mathrm{mg}$ and clopidogrel $75 \mathrm{mg}$ per day was given for 5 days before the procedure. On the procedure day, pigs were anesthetized with zolazepam and tiletamine $\left(2.5 \mathrm{mg} / \mathrm{kg}\right.$, Zoletil50 ${ }^{\circledR}$, Virbac, Caros, France), xylazine (3 mg/kg, Rompun ${ }^{\circledR}$, Bayer AG, Leverkusen, Ger- 
many), and azaperone (6 mg/kg, Stresnil ${ }^{\circledR}$, Janssen-Cilag, Neuss, Germany). They received supplemental oxygen continuously through an oxygen mask. Subcutaneous $2 \%$ lidocaine at the cut-down site was administered, the left carotid artery was surgically exposed, and a 7 French sheath was inserted.

Continuous hemodynamic and surface electrocardiographic monitoring was maintained throughout the procedure. Next, 5,000 units of heparin were intravenously administered as a bolus prior to the procedure, the target coronary artery was engaged using standard 7 F guide catheters, and control angiograms of both coronary arteries were performed using nonionic contrast agent in two orthogonal views.

The stent was deployed by inflating the balloon, obtaining a resulting stent-to-artery ratio of 1.3:1. Coronary angiograms were obtained immediately after stent implantation. Then, all equipment was removed and the carotid artery was ligated. Four weeks after stenting, the animals underwent follow-up angiography in the same orthogonal views before they were sacrificed with an intracoronary injection of $20 \mathrm{~mL}$ potassium chloride.

The hearts were removed and the coronary arteries were pressureperfusion fixed at $110 \mathrm{mmHg}$ in $10 \%$ neutral buffered formalin overnight. All specimens were analyzed by micro- computerized tomography (M-CT) before histopathological testing. Each of the 10 stented arteries in the three groups were step-sectioned, processed routinely for light microscopy, and stained for histological analysis.

\section{Study groups}

The pig was randomly divided into 3 groups: group 1 (sirolimuseluting stent with PLA, SEP, $3.0 \times 16 \mathrm{~mm}, \mathrm{n}=10$ ), group 2 (biolimus A9-eluting stents with PLA, BEP, $3.0 \times 18 \mathrm{~mm}, \mathrm{n}=10$ ), and group 3 (PLA coating stent, PCS, $3.0 \times 16 \mathrm{~mm}, \mathrm{n}=10$ ). A total of 15 pigs were used in this study (15 pigs, 30 coronary arteries, 10 coronary arteries in each group). A SEP, BEP, or PCS was implanted in a randomized manner in the left anterior descending artery and the left circumflex artery of the pigs.

Preparation of PLA-based sirolimus coating on a novel bare-metal stent

In a previous study, our novel Co-Cr alloy BMS (CNUH stent) showed non-inferior histopathological effects compared to a commercial BMS. ${ }^{9}$ PLA was purchased from EVONIK (UK) and sirolimus was purchased LC Laboratories (USA). An ultrasonic spray method was used to apply the coatings to the prepared BMS $(3 \times 16 \mathrm{~mm})$. The required amount of polymer PLA and sirolimus were dissolved in 5 $\mathrm{mL}$ of tetrahydrofuran (THF). The drug solution was then dissolved in the polymer solution. Stents to be coated were cleaned with ethanol and distilled water and then vacuum-dried for $24 \mathrm{hr}$. The sprayed liquid consisted of the polymer/drug system dissolved in THF and diluted to $2 \%$ by weight. The operating requirements in this application called for flow rates of $50 \mu \mathrm{L} / \mathrm{min}$. The stents were placed on a mandrel attached to a rotating shaft. The coated stents were vacuum-dried for $24 \mathrm{hr}$. The surface morphologies of the SEP was examined by scanning electron microscopy (SEM).

The release profiles of sirolimus and biolimus A9 from the prepared SEP and BEP

The release profiles of sirolimus and biolimus A9 from the SEP and $\mathrm{BEP}$, respectively, were acquired using a total buffer extraction method. The sirolimus-coated stent (140 $\mu \mathrm{g} /$ stent) and the biolimus A9coated stent (113 $\mu \mathrm{g} /$ stent) were placed in $3 \mathrm{~mL}$ of PBS at $\mathrm{pH}$ 7.4.
The sample in the buffer was held at $37^{\circ} \mathrm{C}$ while being constantly agitated at $100 \mathrm{rpm}$ in an incubator shaker (JEIO tech, Korea) for 32 days. The buffer in each sample was decanted and replaced with fresh buffer every day. The amount of drug released was determined by the UV-VIS Spectrophotometer at $278 \mathrm{~nm}$. All studies were conducted in triplicates.

Histopathological and micro-computed tomography analysis Histopathologic evaluation of each artery was performed by an experienced cardiovascular pathologist. The specimens were embedded and sections of 50 to $100 \mu \mathrm{m}$ thickness were obtained at about $1 \mathrm{~mm}$ apart and stained with Hematoxylin-Eosin and Carstairs' for histological analysis. Measurements of the histopathologic sections were performed using a calibrated microscope, digital video imaging system, and microcomputer program (Visus 2000 Visual Image Analysis System, IMT Tech). Borders were manually traced for lumen area, the area circumscribed by the internal elastic lamina, and the innermost border of the external elastic lamina (external elastic lamina area). Morphometric analysis of the neointimal area for a given vessel was calculated as the measured internal elastic lamina area minus the lumen area. The measurements were made on five cross-sections from the proximal and distal ends and the three midpoints of each stented segment. Histopathologic stenosis was calculated as $100 \times[1-($ lesion lumen area/lesion internal elastic lamina area) $].{ }^{10}$ The harvested stent specimen was stored in formaldehyde solution. A $1.5 \mathrm{~mL}$ Eppendorf tube was filled with clay, and the clay was tuned with a $\mathrm{V}$ shape to hold the stent during contrast agent staining. The stents were taken from the solution and vertically placed in the V-shaped opening in the clay. Each stent had to be fixed with the clay such that there was no movement of the stent inside the Eppendorf tube. The contrast agent used was omnihexol. One milliliter of the contrast agent was taken in a $5 \mathrm{~mL}$ syringe and injected through the opening at the center of the stent. The stent was incubated with contrast agent overnight and subjected to micro-computed tomography imaging. ${ }^{11}$ All results were interpreted by two independent pathologists in a blind fashion.

Evaluation of arterial injury

Arterial injury at each stent strut site was determined by the anatomic structures penetrated by each stent strut. A numeric value was assigned, as previously described by Schwartz et al. $.^{10}: 0=$ no injury; $1=$ break in the internal elastic membrane; $2=$ perforation of the media; 3 =perforation of the external elastic membrane to the adventitia. The average injury score for each segment was calculated by dividing the sum of the injury scores by the total number of stent struts at the examined section.

Evaluation of inflammation scores, neointimal reaction, and fibrin score

With regard to the inflammation score for each individual stent strut, the grading was as follows: $0=$ no inflammatory cells surrounding the stent strut; $1=$ light, noncircumferential lymphohistiocytic infiltrate surrounding the strut; $2=$ localized, moderate-to-dense cellular aggregate noncircumferentially surrounding the stent strut; $3=$ circumferential dense lymphohistiocytic cell infiltration of the stent strut. The inflammation score for each cross section was calculated by dividing the sum of the individual inflammation scores by the total number of stent strut at the examined section. ${ }^{12}$ Ordinal data for fibrin were collected on each stent section using a scale of 0 to 3 , as previously reported..$^{13}$ 
Statistical analysis

Statistical analysis was performed with the aid of the commercially available software SPSS Version 15 (Chicago, IL, USA). The data were presented as the mean value $\pm \mathrm{SD}$. The unpaired Student's $\mathrm{t}$ test was used for the comparison of the stent groups. Analysis of variance (ANOVA) was used for comparisons of the four stents groups. Ordinal measurements such as injury score, fibrin score, and inflammation score were analyzed using the Mann Whitney and Kruskal-Wallis test. Non-parametric results were presented as medians with interquartile ranges. A value of $P<0.05$ was considered statistically significant.

\section{Results}

Surface morphologies of the SEP

SEM image of the SEP showed a smooth and uniform surface. Bridges and webbings could not be observed in the SEP (Fig. 1).

In vitro cumulative $\%$ release of sirolimus and biolimus A9 by stent

Fig. 2 showed that approximately $40 \%$ of the sirolimus was sustainably released from the stent for 32 days. The BEP indicates a sus-

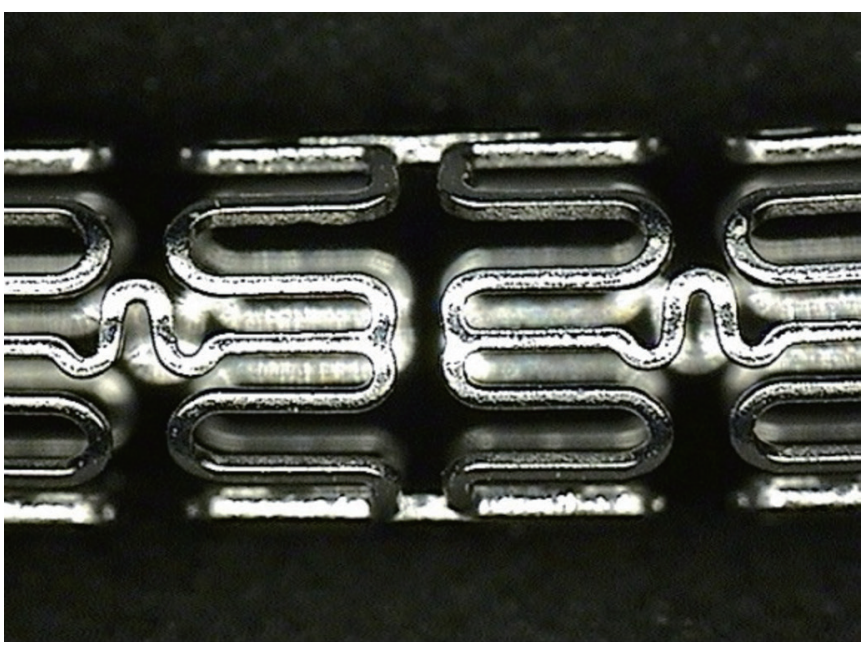

Fig. 1. Surface morphology of the sirolimus-eluting stent with poly-L-lactic acid (SEP) by scanning electron microscopy $(\times 30)$. tained release of $50 \%$ of the biolimus A9 over 5 wk. The drug release kinetics of SEP and BEP were very similar.

Stent implantation

A total of thirty stents (ten SEP, ten BEP, and ten PCS) were placed in the left anterior descending arteries and left circumflex arteries of 15 swine. Mortality for this study was zero. There were no significant differences in the stent-to-artery ratio among the three stent groups. The stent-to-artery ratio was the proportion of the stent diameter divided by the arterial luminal diameter.

Coronary angiographic findings

All in-stent restenosis (ISR) patterns after stenting were ISR pattern II (intra-stent) ${ }^{14}$ in coronary angiographic analysis. ISR pattern II represents restenosis $>10 \mathrm{~mm}$ in length within the stent, without extending outside the stent.

Histopathological analysis among the three groups

There was no significant difference in the injury score [1.0 (range 0.0 to 2.0) in SEP vs. 1.0 (range 0.0 to 2.0) in BEP vs. 1.0 (range 0.0 to 2.0) in PCS, $P=\mathrm{NS}$ ] and the internal elastic lamina $(4.8 \pm 0.68$

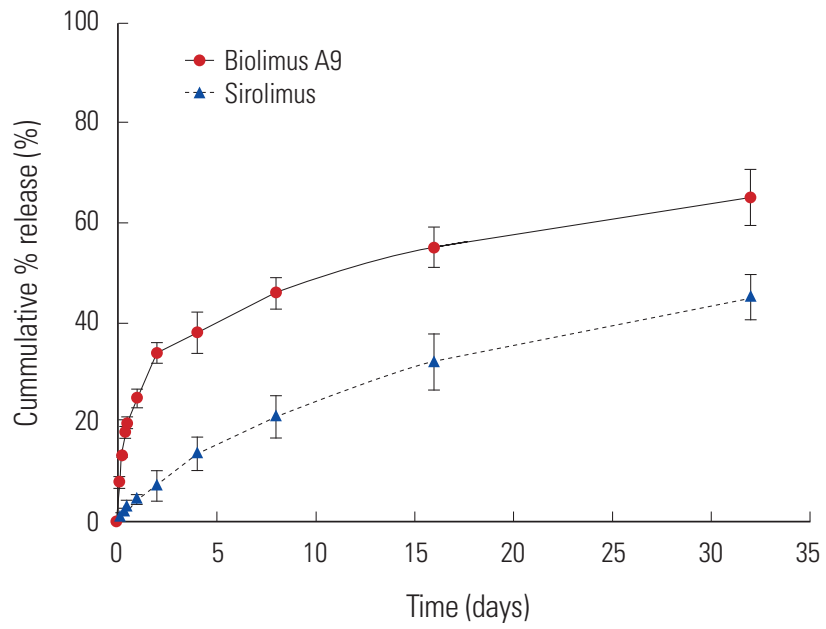

Fig. 2. In vitro cumulative release kinetics of sirolimus from the sirolimuseluting stent with poly-L-lactic acid (SEP) and biolimus A9 from the biolimus A9-eluting stent with poly-L-lactic acid (BEP).
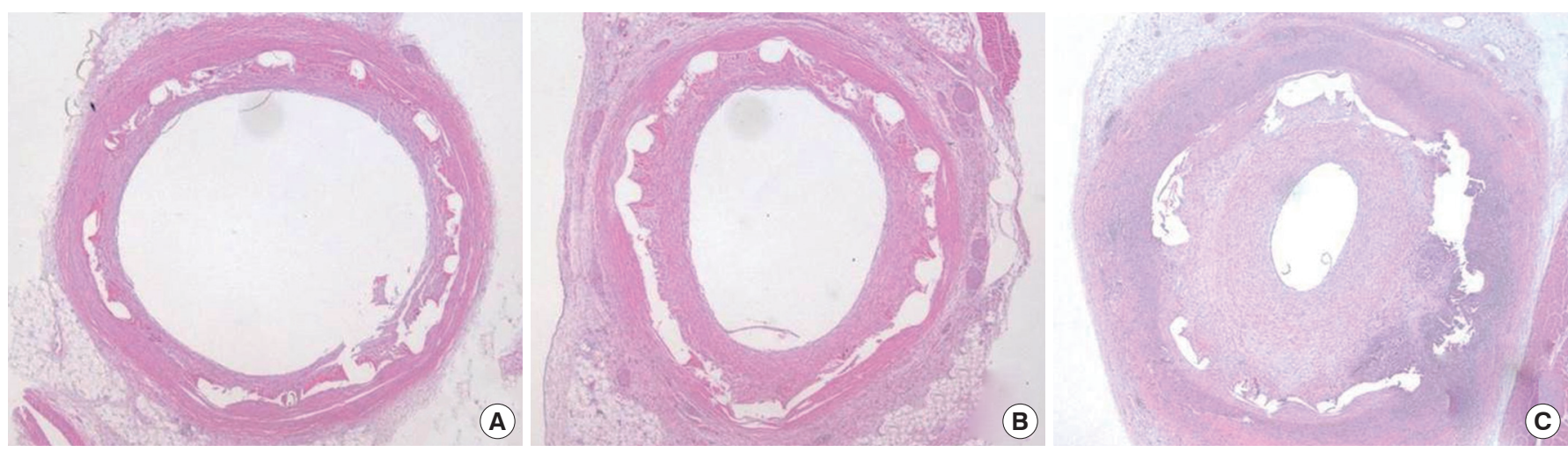

Fig. 3. Representative images of $H \& E$ staining at 4 weeks after stenting. Implanted SEP specimen $(A: \times 20)$, BEP specimen $(B: \times 20)$, and PCS specimen $(C: \times 20)$. SEP, sirolimus-eluting stent with poly-L-lactic acid (PLA); BEP, biolimus A9-eluting stent with PLA; PCS, PLA-coated stent. 
$\mathrm{mm}^{2}$ in SEP vs. $5.0 \pm 0.48 \mathrm{~mm}^{2}$ in BEP vs. $4.8 \pm 0.60 \mathrm{~mm}^{2}$ in PCS, $P=\mathrm{NS})$. There were significant differences in the lumen area $(3.2 \pm$ $0.91 \mathrm{~mm}^{2}$ in SEP vs. $3.1 \pm 1.10 \mathrm{~mm}^{2}$ in BEP vs. $0.5 \pm 0.56 \mathrm{~mm}^{2}$ in PCS, $P<0.0001)$, in the neointimal area $\left(1.7 \pm 0.56 \mathrm{~mm}^{2}\right.$ in SEP vs. $1.9 \pm 0.95 \mathrm{~mm}^{2}$ in BEP vs. $4.3 \pm 0.63 \mathrm{~mm}^{2}$ in PCS, $\left.P<0.0001\right)$, in the percent area of stenosis $(35.2 \pm 13.77 \%$ in SEP vs. $39.0 \pm 20.00 \%$ in BEP vs. $89.4 \pm 10.92 \%$ in PCS, $P<0.0001$ ) (Fig. 3 ), in the fibrin score [2.0 (range 1.0 to 3.0) in SEP vs. 2.0 (range 2.0 to 3.0) in BEP vs. 1.0 (range 1.0 to 2.0 ) in PCS, $P<0.0001$ )] (Fig. 4), and in the inflammation score [1.0 (range 1.0 to 2.0) in SEP vs. 1.0 (range 1.0 to 3.0 ) in BEP vs. 3.0 (range 2.0 to 3.0 ) in PCS, $P<0.0001$ )] among the three groups (Fig. 5).

\section{Micro-computed tomography analysis}

Percent area of stenosis using micro-computed tomography showed similar results with the histopathological analysis $(36.7 \pm 13.77 \%$ in
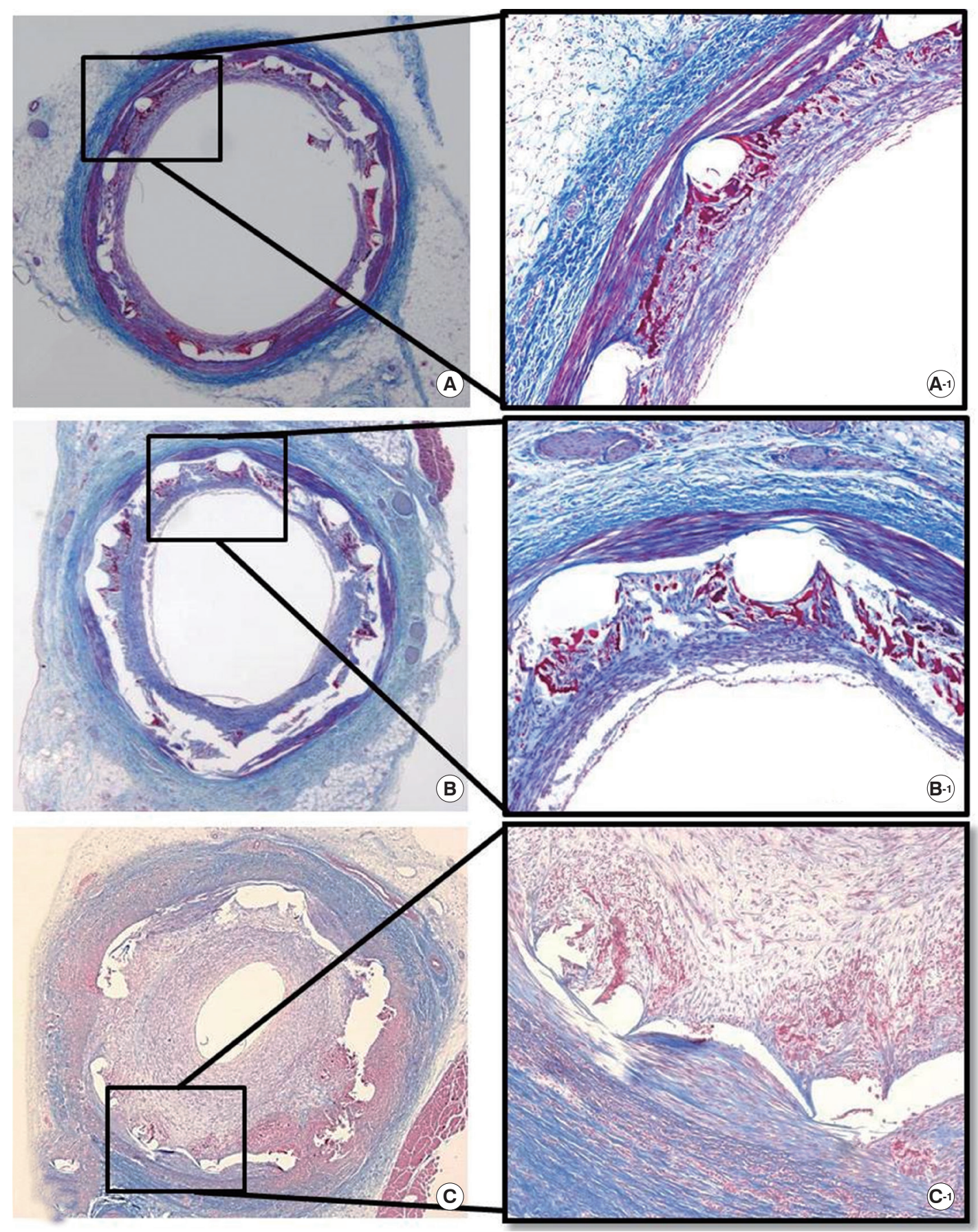

Fig. 4. The Carstair fibrin stain of the low- and high-power fields (magnitude, $\times 20, \times 200$ ) of fibrin infiltration in the implanted SEP $(A, A-1)$, BEP (B, B-1), and PCS (C, C-1).

SEP, sirolimus-eluting stent with poly-L-lactic acid (PLA); BEP, biolimus A9-eluting stent with PLA; PCS, PLA-coated stent. 

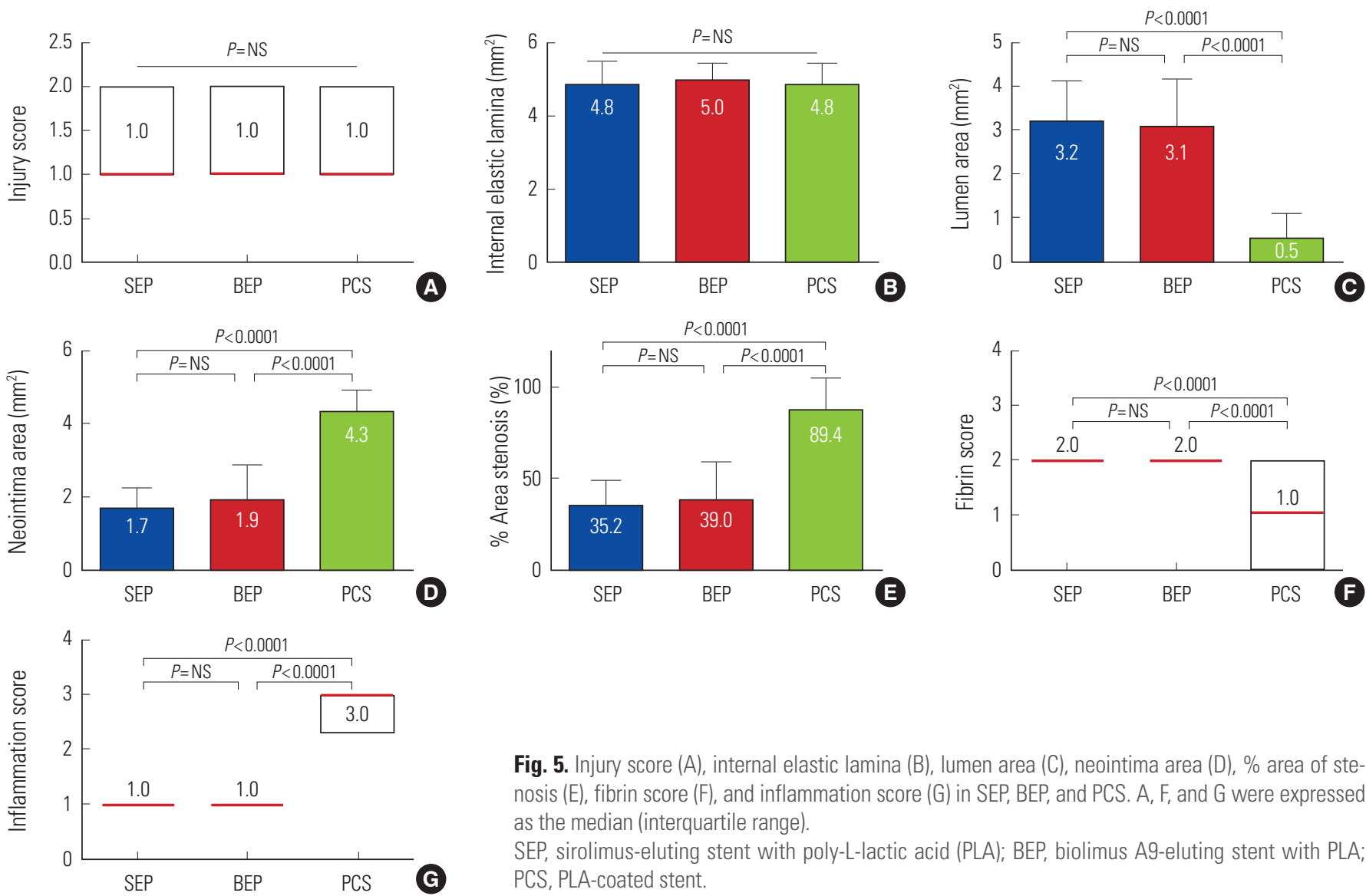

Fig. 5. Injury score (A), internal elastic lamina (B), lumen area (C), neointima area (D), \% area of stenosis $(E)$, fibrin score (F), and inflammation score (G) in SEP, BEP, and PCS. A, F, and G were expressed as the median (interquartile range).

SEP, sirolimus-eluting stent with poly-L-lactic acid (PLA); BEP, biolimus A9-eluting stent with PLA; PCS, PLA-coated stent.

SEP vs. $41.2 \pm 20.00 \%$ in BEP vs. $86.5 \pm 10.92 \%$ in PCS) (Fig. 6).

\section{Discussion}

Our study was conducted to compare a SEP with commercial BEP and PCS in a porcine coronary restenosis model. Our study showed that SEP was not inferior to BEP in its anti-neointimal proliferation effects, and its percent area of stenosis was significantly lower than in PCS.

Sirolimus, also known as rapamycin, has potent antimigratory, antiproliferative, and immumnosuppressive properties and is used to prevent restenosis after coronary artery stenting. Sirolimus is a lipophilic and hydrophobic agent that has low solubility in water. Therefore, the sirolimus that was coated on the coronary stent was easily absorbed by the arterial tissue cells around the stent. ${ }^{15}$ Thus, sirolimus is a suitable agent for coating coronary stents to prevent restenosis.

The first-generation SES with durable polymer are more effective in preventing ISR compared to BMS. ${ }^{16}$ In a porcine coronary restenosis model, a permanent polymer-based SES showed a $50 \%$ reduction in neointima formation and similar degree of endothelialization compared to BMS for 28 days. ${ }^{13}$ In contrast, by 90 days, a SES showed similar neointimal areas and the percent areas of stenosis compared with a control bare metal stent. Moreover, injury and inflammation scores gradually increased between 30 and 180 days. This progression of injury and inflammation induced late neointima formation due to the chronic inflammation of the durable polymer. ${ }^{17}$ Non-bioabsorbable polymers increased the inflammatory reaction when the stents were implanted in porcine coronary arteries. ${ }^{18-20} \mathrm{Se}-$ cond-generation DES, such as zotarolimus-eluting and everolimuseluting stents showed superior clinical outcomes compared to firstgeneration DES. However, second- generation DES also used a permanent polymer for drug coatings.

To overcome the drawbacks of durable polymers, we used bioabsorbable PLA polymers for the stent coating. The PLA polymer is finally converted to water and carbon dioxide by hydrolysis, so it may reduce the long-term side effects of durable polymers. The PLA polymer has been used in many stents such as the BioMatrix ${ }^{\mathrm{TM}}$ stent (Biosensors, BEP) and the NOBORI ${ }^{\mathrm{TM}}$ stent (Terumo Corp.), and its safety and efficacy had been evaluated in clinical trials. ${ }^{21-23}$

Biolimus A9, a sirolimus derivative, is modified rapamycin with an increase in its lipophilicity relative to sirolimus. The modified rapamycin biolimus increases uptake in the stented artery tissue and therefore reduces its distribution in the systemic blood circulation.

BioMatrix $^{\mathrm{TM}}$ was developed by Biosensors as a third-generation DES that elutes biolimus A9 from a bioabsorbable polylactic acid (PLA) polymer. ${ }^{24}$ As previously described, the polymers may be associated with late stent thrombosis, neointima formation, and chronic inflammation. However, Biomatrix ${ }^{\mathrm{TM}}$ releases biolimus A9 into the coronary vessel wall while the PLA polymer decomposes. Therefore, as time passes, Biomatrix ${ }^{\mathrm{TM}}$ significantly reduces the risk of very late stent thrombosis due to polymers.

In our previous study, BEP appeared to be reliable and safe in terms of the arterial inflammatory reaction at overlapping segments compared to non-overlapping segments, and BEP showed better histopathological results than zotarolimus- and everolimus-eluting 


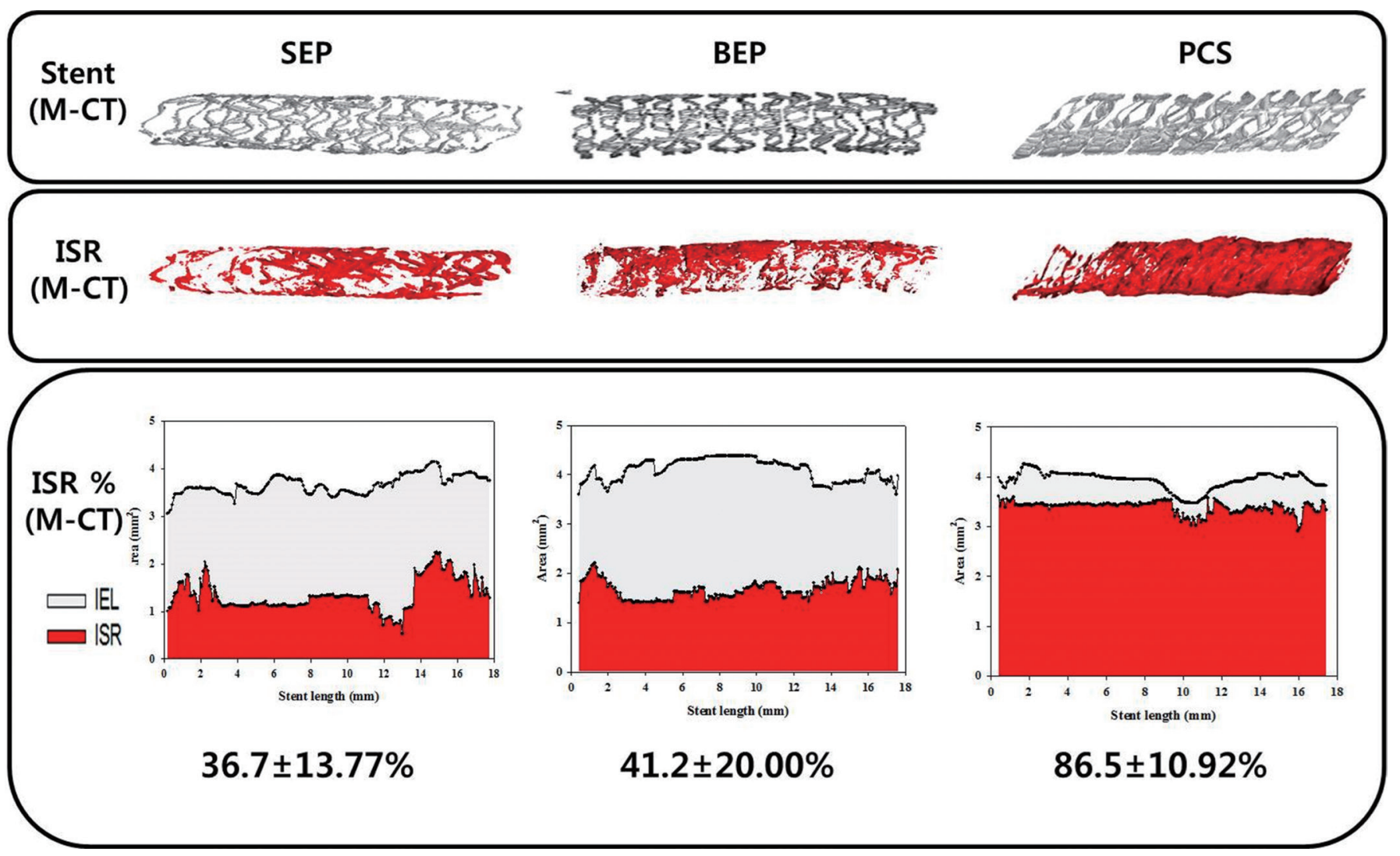

Fig. 6. Micro-computed tomography analysis of in-stent restenosis in the SEP, BEP, and PCS.

SEP, sirolimus-eluting stent with poly-L-lactic acid (PLA); BEP, biolimus A9-eluting stent with PLA; PCS, PLA-coated stent; ISR, in-stent restenosis; IEL, internal elastic lamina.

stents in a porcine coronary restenosis model. ${ }^{25,26}$ In clinical research, BES demonstrated a lower rate of the composite of major adverse cardiac events in patients with ST-segment elevation myocardial infarction undergoing primary percutaneous coronary intervention. ${ }^{27}$

However, to date, there is no preclinical data on the efficacy of sirolimus-eluting stents with biodegradable PLA polymers compared with commercial stents with PLA. Our study showed that SEP and commercial BEP showed similar and superior neointima suppressive effects compared to PCS that did not use sirolimus derivatives. Currently, sirolimus is not used in coronary stent coating, unlike other -limus derivatives such as biolimus A9, everolimus, and zotrarolimus. However, our results demonstrated that sirolimus was not inferior to the recently-developed biolimus A9.

SEP and BEP showed superior and similar neointima suppressive effects compared to PLA polymer-coated stents at one month after stenting in a porcine coronary restenosis model. Therefore, sirolimus with biodegradable polymers can be a useful and selective alternative drug for coronary stent coating to inhibit neointimal hyperplasia.

\section{Acknowledgments}

This study was supported by a grant from the Korean Health Technology R\&D Project (HI13C1527), Ministry of Health \& Welfare, Republic of Korea, the Cardiovascular Research Center, Chonnam National University Hospital, and the Regeneromics Research center, Chonnam National University.

\section{Conflicts of Interest}

There are no conflicts of interest relevant to this article to report.

\section{References}

1. Stettler C, Wandel S, Allemann S, Kastrati A, Morice $\mathrm{MC}$, Schomig A, et al. Outcomes associated with drugeluting and bare-metal stents: a collaborative network meta-analysis. Lancet 2007;370:937-48.

2. Stone GW, Moses JW, Ellis SG, Schofer J, Dawkins $\mathrm{KD}$, Morice MC, et al. Safety and efficacy of sirolimus- and paclitaxel-eluting coronary stents. N Engl J Med 2007;356:998-1008

3. Joner M, Finn AV, Farb A, Mont EK, Kolodgie FD, La- dich E, et al. Pathology of drug-eluting stents in humans: delayed healing and late thrombotic risk. J Am Coll Cardiol 2006;48:193-202.

4. Cook S, Ladich E, Nakazawa G, Eshtehardi P, Neidhart $\mathrm{M}$, Vogel R, et al. Correlation of intravascular ultrasound findings with histopathological analysis of thrombus aspirates in patients with very late drug-eluting stent thrombosis. Circulation 2009;120:391-9.

5. Nakazawa G, Finn AV, Joner M, Ladich E, Kutys R, Mont EK, et al. Delayed arterial healing and increased late stent thrombosis at culprit sites after drug-eluting stent placement for acute myocardial infarction patients: an autopsy study. Circulation 2008;118:1138-45

6. Rossi F, Casalini T, Raffa E, Masi M, Perale G. Bioresorbable polymer coated drug eluting stent: a model study. Mol Pharm 2012;9:1898-910.

7. Tamai H, Igaki K, Kyo E, Kosuga K, Kawashima A, Matsui S, et al. Initial and 6-month results of biodegradable poly-1-lactic acid coronary stents in humans. Circulation 2000;102:399-404.

8. Nishio S, Kosuga K, Igaki K, Okada M, Kyo E, Tsuji T, et al. Long-term ( $>10$ Years) clinical outcomes of firstin-human biodegradable poly-l-lactic acid coronary stents: Igaki-Tamai stents. Circulation 2012;125: 2343-53. 
9. Lim KS, Bae IH, Kim JH, Park DS, Kim JM, Sim DS, et al. Mechanical and histopathological comparison between commercialized and newly designed coronary bare metal stents in a porcine coronary restenosis model. Chonnam Med J 2013;49:7-13.

10. Schwartz RS, Huber KC, Murphy JG, Edwards WD, Camrud AR, Vlietstra RE, et al. Restenosis and the proportional neointimal response to coronary artery injury: results in a porcine model. J Am Coll Cardiol 1992;19: 267-74.

11. Che HL, Bae IH, Lim KS, Song IT, Lee H, Muthiah M, et al. Suppression of post-angioplasty restenosis with an Akt1 siRNA-embedded coronary stent in a rabbit model. Biomaterials 2012;33:8548-56.

12. Schwartz RS, Edelman E, Virmani R, Carter A, Granada JF, Kaluza GL, et al. Drug-eluting stents in preclinical studies: updated consensus recommendations for preclinical evaluation. Circ Cardiovasc Interv 2008; 143-53.

13. Suzuki T, Kopia G, Hayashi S, Bailey LR, Llanos G Wilensky R, et al. Stent-based delivery of sirolimus reduces neointimal formation in a porcine coronary model. Circulation 2001;104:1188-93.

14. Mehran R, Dangas G, Abizaid AS, Mintz GS, Lansky AJ, Satler LF, et al. Angiographic patterns of in-stent restenosis: classification and implications for long-term outcome. Circulation 1999;100:1872-8.

15. Gummert JF, Ikonen T, Morris RE. Newer immunosuppressive drugs: a review. J Am Soc Nephrol 1999;10: 1366-80.
16. Park SJ, Kim YH, Lee BK, Lee SW, Lee CW, Hong MK, et al. Sirolimus-eluting stent implantation for unprotected left main coronary artery stenosis: comparison with bare metal stent implantation. J Am Coll Cardiol 2005;45:351-6.

17. Carter AJ, Aggarwal M, Kopia GA, Tio F, Tsao PS, Kolata $\mathrm{R}$, et al. Long-term effects of polymer-based, slowrelease, sirolimus-eluting stents in a porcine coronary model. Cardiovasc Res 2004;63:617-24.

18. van Beusekom HM, Schwartz RS, van der Giessen WJ. Synthetic polymers. Semin Interv Cardiol 1998;3:145-8.

19. van der Giessen WJ, Lincoff AM, Schwartz RS, van Beusekom HM, Serruys PW, Holmes DR, Jr., et al. Marked inflammatory sequelae to implantation of biodegradable and nonbiodegradable polymers in porcine coronary arteries. Circulation 1996;94:1690-7.

20. van Beusekom HM, Serruys PW, van der Giessen WJ. Coronary stent coatings. Coron Artery Dis 1994;5:590-6.

21. Cassese S, Fusaro M, Byrne RA, Tada T, Hoppmann P, Joner M, et al. Clinical outcomes of patients treated with Nobori biolimus-eluting stent: meta-analysis of randomized trials. Int J Cardiol 2014;175:484-91.

22. Sabate M, Raber L, Heg D, Brugaletta S, Kelbaek H, Cequier A, et al. Comparison of newer-generation drugeluting with bare-metal stents in patients with acute ST-segment elevation myocardial infarction: a pooled analysis of the EXAMINATION (clinical Evaluation of the Xience-V stent in Acute Myocardial INfArcTION) and COMFORTABLE-AMI (Comparison of Biolimus Eluted From an Erodible Stent Coating With
Bare Metal Stents in Acute ST-Elevation Myocardial Infarction) trials. JACC Cardiovasc Interv 2014;7:55 63.

23. Palmerini T, Biondi-Zoccai G, Della Riva D, Mariani A Sabate M, Smits PC, et al. Clinical outcomes with bioabsorbable polymer- versus durable polymer-based drugeluting and bare-metal stents: evidence from a comprehensive network meta-analysis. J Am Coll Cardiol 2014 63:299-307.

24. Grube E, Buellesfeld L. BioMatrix Biolimus A9-eluting coronary stent: a next-generation drug-eluting stent for coronary artery disease. Expert Rev Med Devices 2006;3:731-41.

25. Park KH, Jeong MH, Kim JM, Park DS, Kim JH, Lim $\mathrm{KS}$, et al. The impact of triple anti-platelet therapy for endothelialization and inflammatory response at overlapping bioabsorbable polymer coated drug-eluting stents in a porcine coronary model. Int J Cardiol 2013;168: 1853-8.

26. Lim KS, Jeong MH, Bae IH, Park DS, Kim JM, Kim $\mathrm{JH}$, et al. Histopathological Comparison among Biolimus, Zotarolimus and Everolimus-Eluting Stents in Porcine Coronary Restenosis Model. Korean Circ J 2013;43: 744-51.

27. Raber L, Kelbaek H, Ostoijc M, Baumbach A, Heg D, Tuller D, et al. Effect of biolimus-eluting stents with biodegradable polymer vs bare-metal stents on cardiovascular events among patients with acute myocardia infarction: the COMFORTABLE AMI randomized trial. JAMA 2012;308:777-87. 